compared between those patients with and without co-morbidities (Charlson index 1 or more vs 0 ) using unpaired t-test or non-parametric equivalent.

Results 84 patients (73\%) had a Charlson index of 1 or more. No difference in age, gender, FEV1, or MRC Dyspnoea score was seen between those with and without co-morbidities. Mean (SD) change in ISW following PR was not significantly different between those with and without co-morbidities (63 (96) vs 33 (88); $p=0.13$ ). Similarly there were no significant differences in CRQ-D change (4.1 (6.4) vs 5.7 (7.1); $\mathrm{p}=0.26$ ), CRQ-F change (2.6 (5.1) vs 4.4 (5.7); $\mathrm{p}=0.13)$, CRQ-E change (3.6 (7.8) vs $6.4(7.7) ; \mathrm{p}=0.09)$ or CRQ-M change $(2.7$ (4.8) vs $2.3(6.9) ; p=0.75)$. No association was seen between Charlson index and change in ISW following PR (Spearman rank=0.09; $\mathrm{p}=0.32$ ).

Discussion The prevalence of co-morbidities in COPD patients undergoing PR is high. The presence of co-morbidities does not seem to affect patients' response to pulmonary rehabilitation.

\section{Changing patterns of mycobacterial disease P54 DO THE NICE NEW ENTRANT TB SCREENING GUIDELINES
UNDER-DIAGNOSE CASES OF LATENT TB INFECTION?}

doi:10.1136/thx.2010.150979.5

D Thomas, M Jarvis, A Williams. The Royal Bournemouth and Christchurch Hospitals NHS Foundation Trust, Bournemouth, UK

Introduction In 2006, $72 \%$ of active TB cases in the UK occurred in people born overseas (HPA 2008). 48\% of new entrants with TB were diagnosed within 5 years of entering the UK and 19\% within 2 years (HPA 2008). It is a priority therefore, to identify and appropriately treat those infected with latent $\mathrm{TB}$ infection through TB screening programmes (DH 2004). NICE (2006) TB new entrant screening guidelines allow certain groups of new entrants to be screened solely via chest $\mathrm{x}$-ray (CXR), omitting a Tuberculin Skin Test (TST). This potentially under-diagnoses latent TB Infection (LTBI). The aim of this study was to determine whether NICE (2006) criteria are adequate in detecting latent TB.

Method A retrospective case-note analysis of new entrants over a 44month period (2006-2009). All patients were screened using a locally developed 'Dorset' algorithm that combined CXR and TST unless contraindicated (see Abstract P54 Figure 1). Each case was then re-evaluated using the NICE algorithm. This allowed direct comparison of each algorithm to detect LTBI.

Results 547 new entrants were referred locally for TB screening (2006-2009). 397 attended. 41 (10.3\%) patients (all HIV-ve) were diagnosed with LTBI, based on the following outcomes:

- Abnormal CXR and strongly positive TST=14 (34\%).

- Normal CXR but strongly positive TST=18 (44\%).

- Abnormal CXR but normal TST=9 (22\%).

Comparison of the two algorithms showed that while all 41 cases were detected using the Dorset algorithm, only 27 cases (65.8\%) were detected using the NICE algorithm. This represents a $34.1 \%$ shortfall in LTBI detection using NICE (95\% CI 19.63\% to $48.67 \%$, $99 \%$ CI $15.04 \%$ to $53.26 \%$ ).

Discussion This study demonstrated that through the omission of TST, the NICE algorithm missed 14 (34.1\%) cases of LTBI compared with the Dorset algorithm. While alternative screening methods such as IGRA are increasing in recognition, these continue to be an expensive option if not provided locally. Therefore TB services without routine access to IGRA can significantly improve their detection of latent TB by simply combining their existing screening tools.

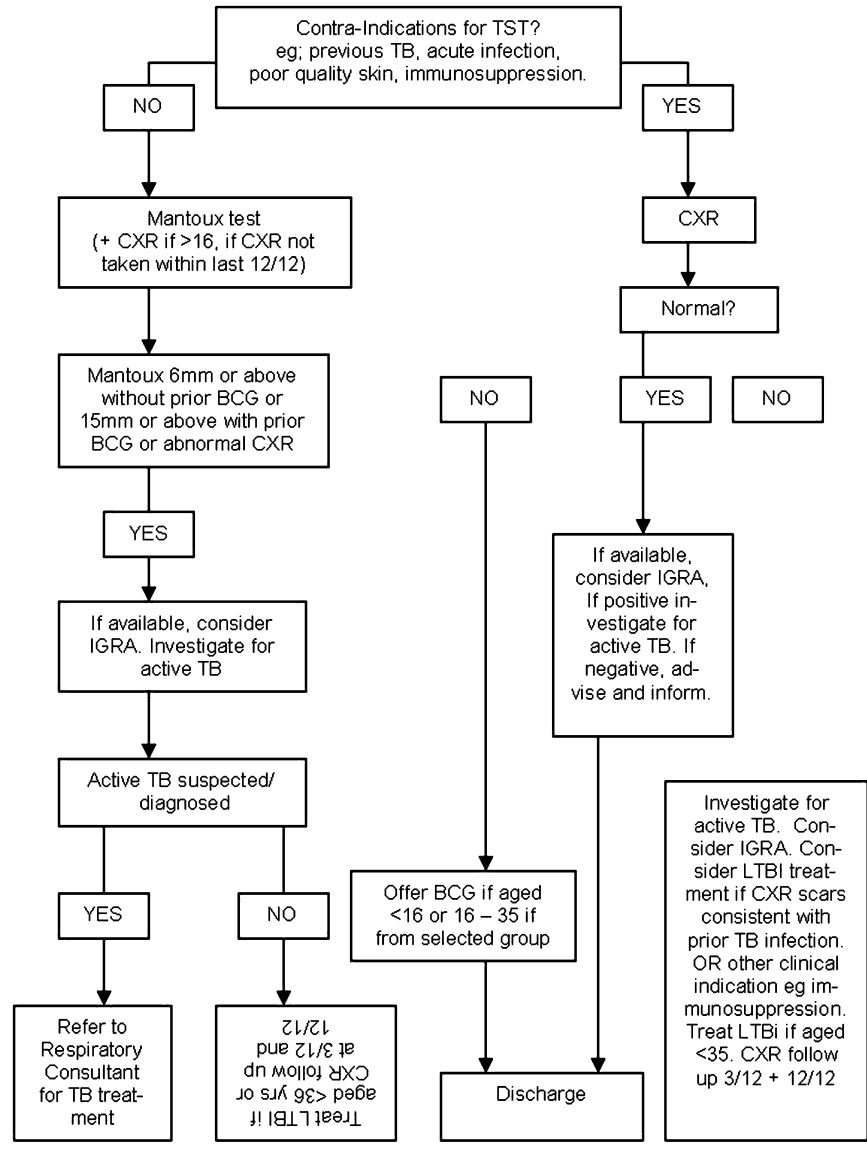

'Inform and advise' information should be offered to all New Entrants.

Treatment for LTBI should be offered to those aged $<35$ and any age of health care worker or HIV +ve individual.

Selected group - from sub-Saharan Africa or a country with TB incidence $>500 / 100,000$

Abstract P54 Figure 1 Dorset new entrant TB screening algorithm (2009).

\section{P55 POST-BRONCHOSCOPY SPUTUM: INCREASING THE DIAGNOSTIC YIELD IN SMEAR NEGATIVE PULMONARY TUBERCULOSIS}

doi:10.1136/thx.2010.150979.6

${ }^{1} \mathrm{P}$ M George, ${ }^{1} \mathrm{M}$ Mehta, ${ }^{1} \mathrm{~J}$ Dhariwal, ${ }^{1} \mathrm{~A}$ Singanayagam, ${ }^{1} \mathrm{C}$ E Raphael, ${ }^{1} \mathrm{M}$ Salmasi, ${ }^{1} \mathrm{D}$ W Connell, ${ }^{1} \mathrm{P}$ Molyneaux, ${ }^{1} \mathrm{M}$ Wickremasinghe, ${ }^{2} \mathrm{~A}$ Jepson, ${ }^{1} \mathrm{O} \mathrm{M}$ Kon. ${ }^{1}$ Chest and Allergy Department, St Mary's Hospital, Imperial College Healthcare NHS Trust, London, UK; ${ }^{2}$ Department of Microbiology, St Mary's Hospital, Imperial College Healthcare NHS Trust, London, UK

Background The prevalence of smear negative pulmonary tuberculosis (PTB) is increasing. At many centers, active PTB suspects who are Acid-Fast Bacilli (AFB) smear negative or non-productive of sputum undergo fiber optic bronchoscopy for bronchoalveolar lavage but post bronchoscopy sputum (PBS) sampling is not routine. The aim of the study was to establish the clinical utility of PBS sampling in this subgroup of patients with active PTB.

Methods A retrospective study of all patients attending a central London University hospital with microbiologically confirmed PTB between January 2004 and December 2009. Patients who were AFB smear negative or non-productive of sputum were eligible for the study if a sputum sample was obtained within 7 days of bronchoscopy.

Results The cohort $(\mathrm{n}=50)$ was heterogeneous - 29 were male (58\%), 12 were infected with HIV (24\%), 19 were of African origin (38\%), 17 were white Caucasian $(34 \%)$ and four were from the 